\title{
BMJ Open Protocol for a systematic review of economic evaluations of preoperative smoking cessation interventions for preventing surgical complications
}

\author{
Nikki McCaffrey (D) , ${ }^{1}$ Julie Higgins, ${ }^{2}$ Anita Lal ${ }^{1}$
}

To cite: McCaffrey N, Higgins J, Lal A. Protocol for a systematic review of economic evaluations of preoperative smoking cessation interventions for preventing surgical complications. BMJ Open 2021;11:e057171. doi:10.1136/ bmjopen-2021-057171

- Prepublication history and additional supplemental material for this paper are available online. To view these files, please visit the journal online (http://dx.doi.org/10.1136/ bmjopen-2021-057171).

Received 06 September 2021 Accepted 20 October 2021

Check for updates

(C) Author(s) (or their employer(s)) 2021. Re-use permitted under CC BY-NC. No commercial re-use. See rights and permissions. Published by BMJ.

${ }^{1}$ Deakin Health Economics, Deakin University School of Health and Social Development, Burwood, Victoria, Australia ${ }^{2}$ Health, Deakin University Library, Burwood, Victoria, Australia

Correspondence to Dr Nikki McCaffrey; nikki.mccaffrey@deakin.edu.au

\section{ABSTRACT}

Introduction The short-term economic benefit of embedding best practice tobacco dependence treatment (TDT) into healthcare services prior to surgery across different populations and jurisdictions is largely unknown. The aim of this systematic review is to summarise the cost-effectiveness of preoperative smoking cessation interventions for preventing surgical complications compared with usual care. The results will provide hospital managers, clinicians, healthcare professionals and policymakers with a critical summary of the economic evidence on providing TDT routinely before surgery, aiding the development and dissemination of unified, best practice guidelines, that is, implementation of article 14 of the WHO Framework Convention on Tobacco Control.

Methods and analysis A comprehensive search of peer-reviewed literature will be conducted from database inception until 23 June 2021 (Cochrane, Econlit, Embase, Health Technology Assessment, Medline Complete, Scopus). Published, English-language articles describing economic evaluations of preoperative smoking cessation interventions for preventing surgical complications will be included. One researcher will complete the searches and two researchers will independently screen results for eligible studies. Any disagreement will be resolved by the third researcher. A narrative summary of included studies will be provided. Study characteristics, economic evaluation methods and cost-effectiveness results will be extracted by one reviewer and descriptive analyses will be undertaken. A second reviewer will review data extracted for accuracy from $10 \%$ of the included studies. Reporting and methodological quality of the included studies will be evaluated independently by two reviewers using the Consolidated Health Economic Evaluation Reporting Standards statement and the Quality of Health Economic Studies Instrument checklist, respectively.

Ethics and dissemination This research does not require ethics approval because the study is a planned systematic review of published literature. Findings will be presented at health economic, public health and tobacco control conferences, published in a peer-reviewed journal and disseminated via social media.

Trial registration number CRD42021257740.

\section{Strengths and limitations of this study}

- This review will, for the first time, summarise and assess the evidence on the cost-effectiveness of preoperative smoking cessation interventions for preventing complications compared with usual care across different surgical populations.

- The search strategy was developed by an experienced health liaison librarian.

- The systematic review is registered with the International Prospective Register of Systematic Reviews and the protocol is reported according to the recommendations of the Preferred Reporting Items for Systematic Review and Meta-Analysis Protocols statement.

- Two authors will independently screen the search results and assess the reporting and methodological quality of the included studies.

- The total number of studies evaluating the costeffectiveness of preoperative smoking cessation interventions for preventing surgical complications may be small; economic evaluation methods may be heterogeneous; and cost-effectiveness estimates may be derived from diverse health financing systems, limiting the generalisability of findings.

\section{INTRODUCTION}

Despite substantial progress in the reduction of tobacco use, smoking remains a leading preventable cause of premature mortality and morbidity globally. ${ }^{1-3}$ In 2003, the WHO established the WHO Framework Convention on Tobacco Control (FCTC), an international treaty promoting the implementation of evidence-based measures to reduce tobacco use and exposure to tobacco smoke. ${ }^{4}$ Signatories are obligated, among other things, to provide treatment for tobacco dependence as set out in article $14 .{ }^{5}$ However, best practice tobacco dependence treatment (TDT) combining approved pharmacotherapy with multisession behavioural support-is not systematically embedded in the healthcare system of some countries such as Australia. ${ }^{5-7}$ 
A hospital admission provides a 'teachable moment' for health education and smoking cessation counselling, particularly for surgical patients. ${ }^{8}{ }^{9}$ Despite this, hospital policies on the provision of TDT vary across jurisdictions and hospitals. ${ }^{10-12}$ Those who smoke are at higher risk of surgical complications with significantly higher odds of complications leading to further surgery and readmission compared with non-smokers $(15 \%$ vs $12 \%$, respectively) ${ }^{6}{ }^{13}$ Smokers are also at higher risk of poor postoperative healing compared with non-smokers including surgical site infections (SSIs) (OR 1.79, 95\% CI 1.57 to 2.04), wound complications (OR 2.27, $95 \%$ CI 1.82 to 2.84 ) and delayed healing and wound separation (OR 2.07, 95\% 1.53 to 2.81). ${ }^{14}$ Postsurgical complications increase morbidity and mortality, reduce quality of life, prolong hospitalisation, increase healthcare costs and result in lost productivity. ${ }^{61516}$

Quitting smoking significantly improves surgical outcomes, shortens hospital stays and reduces cancer recurrences and deaths. ${ }^{17}$ Abstaining from smoking just 4 weeks before surgery significantly reduces the risk of complications such as SSIs and pulmonary complications. ${ }^{15}$ Undergoing surgery can provide motivation to quit smoking ${ }^{8}$ and interventions delivered presurgery increase the likelihood of success. ${ }^{1819}$

There is a substantial body of evidence on the longterm costs and benefits of TDT ${ }^{20-23}$ but the health and economic benefits of routinely providing smoking cessation in the shorter term are less well known. A recent cost analysis suggests, all else being equal, reducing the 2016 maternal smoking rate from $8.4 \%$ to $6.4 \%$ could have saved 106 Victorian public hospital neonatal intensive care cot-days $(\$ A 276000)^{24}$ in 1 year. Further, 3580 (95\% uncertainty interval (UI) 2312 to 5178) SSIs could have been prevented, and 8985 (95\% UI 4094 to 19 $153)$ hospital bed-days and $\$ \mathrm{~A} 19.1 \mathrm{M}(95 \%$ UI $\$ \mathrm{~A} 7.7 \mathrm{M}$ to $\$ \mathrm{~A} 42.5 \mathrm{M}$ ) saved in Australia if the 2016 surgical smoking rate had reduced from $23.9 \%$ to $10 \% .{ }^{25}$ However, these analyses only partially consider the range of costs and benefits associated with increasing smoking cessation rates. For example, the costs of providing TDT are not included in the estimates.

Economic evaluations systematically compare the costs and benefits of competing interventions and thus provide information on how best to improve outcomes within funding constraints. ${ }^{26} 27$ Targeted and accessible economic information for hospital decision-makers, about the costs and benefits of routinely providing TDT for surgical populations could help promote the implementation of evidence-based practice in hospitals. ${ }^{28}$ Jiménez-Ruiz et al recently estimated for every Euro invested in providing smoking cessation before surgery in Spain returns an estimated $€ 1.29 .^{29}$ Thus far, there is no systematic review providing an overview of the cost-effectiveness of preoperative smoking cessation to prevent complications in different surgical populations and jurisdictions to inform service providers, clinicians, funders and policymakers. Consequently, the aim of this systematic review is to summarise and assess the evidence on the costs and benefits of preoperative smoking cessation interventions for preventing surgical complications compared with usual care across different populations. The findings will help inform the development of appropriate policies, programmes and strategies for embedding TDT in hospital settings, assisting the implementation of article 14 of the WHO FCTC.

\section{Review questions}

This systematic review addresses three related research questions:

1. What is the cost-effectiveness of preoperative smoking cessation interventions for preventing surgical complications versus usual care across different populations and jurisdictions?

2. Which preoperative smoking cessation interventions are the most cost-effective versus usual care?

3 . What is the reporting and methodological quality of peer-reviewed, published health economic evaluations of preoperative smoking cessation interventions for preventing surgical complications?

\section{METHODS AND ANALYSIS}

The protocol is reported in accordance with the Preferred Reporting Items for Systematic Reviews and Meta-Analyses (PRISMA) statement. ${ }^{30}$

\section{Eligibility criteria}

The eligibility criteria are summarised in table 1 . Published, peer-reviewed, English-language articles reporting full economic evaluations of preoperative smoking cessation to prevent complications in surgical populations will be eligible for inclusion in the systematic review. Health services costing studies, partial economic evaluations, editorials, comment or discussion papers, qualitative studies, reviews, case reports, case series, book chapters and conference articles will be excluded.

\section{Search strategy}

The search strategy will be devised, tested and adapted for the different databases by the Deakin University Health Liaison Librarian $(\mathrm{JH})$ in conjunction with the lead researcher (NMC). Search terms, that is, key words and subject headings, will be based on the research question, population (smokers), intervention type (preoperative), study design (economic evaluation), outcome (complications) and previous systematic reviews of preoperative smoking cessation interventions to prevent surgical complications. ${ }^{14} 173132$

A search will be conducted from database inception until 23 June 2021 in the following electronic databases: Cochrane library; Econlit; EMBASE; Health Technology Assessment; Medline Complete; and Scopus. The databases were chosen based on the experience of the librarian and the recommendations by Arber $e t a l^{33} \mathrm{~A}$ scoping search of the grey literature will be conducted using Google search engine to determine whether a more 


\begin{tabular}{|c|c|c|}
\hline PICOS & Inclusion criteria & Exclusion criteria \\
\hline Population & $\begin{array}{l}\text { Adult patients* from any jurisdiction undergoing any } \\
\text { type of elective surgery and are current smokers }\end{array}$ & Patients undergoing emergency surgery \\
\hline Intervention & $\begin{array}{l}\text { All smoking cessation interventions, including brief } \\
\text { advice, behavioural support, pharmacotherapy } \\
\text { (nicotine replacement therapy, varenicline, bupropion), } \\
\text { individually or in combination. }\end{array}$ & $\begin{array}{l}\text { Details of smoking cessation intervention not } \\
\text { provided, for example, brief advice plus nicotine } \\
\text { replacement therapy (NRT) where the type of NRT is } \\
\text { not described. }\end{array}$ \\
\hline Control & Usual care & $\begin{array}{l}\text { No usual care comparator, for example, compares } \\
\text { alternative smoking cessation interventions only }\end{array}$ \\
\hline Outcome & $\begin{array}{l}\text { Surgical complications } \dagger \\
\text { Incremental cost-effectiveness ratio }\end{array}$ & No costing data provided. \\
\hline Study type & $\begin{array}{l}\text { Cost-benefit analysis, cost-utility analysis, cost- } \\
\text { effectiveness analysis, cost-consequences analysis. } \\
\text { Trial-based or modelled analyses. }\end{array}$ & $\begin{array}{l}\text { Health services costing studies, partial economic } \\
\text { evaluations, editorials, comment or discussion } \\
\text { papers, qualitative studies, reviews, case reports, } \\
\text { case series, book chapters and conference articles. }\end{array}$ \\
\hline
\end{tabular}

*18 years and older.

†Complications may include necrosis, healing delay and dehiscence, surgical site infection, wound complications, pulmonary complications, hernia, lack of fistula or bone healing, readmission or mortality.

comprehensive search of the grey literature is warranted. Backwards and forwards citation tracing of included articles will be conducted for additional literature unidentified by the search. Search results will be stored in Thomson Reuters EndNote V. X9.2 (2019) libraries and Covidence software ${ }^{34}$ will be used to manage records throughout the review. The final search strategies are presented in the online supplemental file.

\section{Selection process}

The titles and abstracts of the studies identified by the search will be screened independently by two researchers (NMC, AL) to determine eligibility for inclusion in the review. Full text articles will be retrieved for assessment when the abstract contains insufficient information. Subsequently, full-text articles for all potentially eligible records will be independently screened for inclusion in the review (NMC, AL) and reasons for exclusion will be recorded. Any disagreement will be resolved by the third researcher $(\mathrm{JH})$. Results of the study selection process will be presented as a the PRISMA flow diagram and a list of excluded articles will be provided.

\section{Risk of bias and quality appraisal}

Methodological quality of the included studies will be evaluated independently by two researchers (NMC, AL) using the Quality of Health Economic Studies Instrument (QHES) checklist, one of the most widely applied economic evaluation checklists. ${ }^{35-37}$ The QHES consists of sixteen 'yes/no' questions with each question assigned a weight based on importance and total scores range from 0 (poorest quality) to 100 (highest quality). Further, the QHES is a validated checklist with test-retest reliability. ${ }^{35}$ Reporting quality of the included studies will also be evaluated independently by the two researchers using the commonly applied Consolidated Health Economic Evaluation Reporting Standards statement. ${ }^{38}$ Any disagreement will be resolved by consensus among the team.

\section{Data collection}

A data extraction form will be developed in Excel based on previously reported systematic reviews of economic evaluations and will be piloted with two included studies by two reviewers (NMC, AL) and updated if necessary. Bibliographic (lead author, publication date, country), study (patient population, intervention, timing intervention commenced, comparator, outcome measures) and methodological (type of analysis, design, time horizon, perspective, reference year, currency, discount rate, resource use, costs, statistical analysis, methods of handling missing data, incremental cost-effectiveness ratio (ICER), uncertainty analysis) information will be extracted. One researcher (NMC) will extract the data from the included studies and a second researcher (AL) will check the data extraction. The views of the third researcher $(\mathrm{JH})$ will be sought where there is ambiguity or disagreement.

\section{Data synthesis}

A critical, narrative summary of the included studies will be provided commensurate with guidance from the Cochrane Collaboration. ${ }^{40}$ Characteristics and findings of the included studies will be summarised and presented for different types of surgery, different periods of time the preoperative intervention was commenced and alternative outcomes (where feasible). All relevant studies will be included in the review, that is, no study will be excluded on quality criteria, although an assessment of how the quality of the studies may affect the main results and outcome measures will be presented. The methodologies of the included studies will summarised and compared, together with the quality appraisals and risk of bias assessments. A meta-analysis will not be conducted as economic 
evaluations are typically heterogenous. ${ }^{41} 42$ The ICERs will be converted to 2021 Euros using the web-based tool developed by the Cochrane Campbell Economic Methods Group (CCEMG) and the Evidence for Policy and Practice Information and Coordinating Centre (EPPI-Centre) Cost Converter. ${ }^{43} 44$

\section{Patient and public involvement}

A consumer advisory panel of six members, representing broad and objective consumer perspectives, provided input into the development of the research programme which includes this review.

\section{Ethics and dissemination}

Ethics approval to conduct this research is not required because this study is a planned systematic review of published economic literature. The systematic review protocol is registered with the PROSPERO, registration number CRD42021257740. Findings will be presented at leading tobacco control and health economic conferences, published in a peer-reviewed journal and disseminated via website postings such as the Deakin University Institute for Health Transformation Linkedin website and social media channels such as Twitter (eg, @DHE_ Deakin, @IHT_Deakin) and Facebook.

Acknowledgements Many thanks to the CAP for their continued input into the research program, "Economics of Cancer Care across the Continuum in Victoria (ECCC)" (https://www.victoriancanceragency.vic.gov.au/2020-funding-recipients).

Contributors NMC led the conception and design of the work with input from $\mathrm{JH}$. NMC drafted the work and AL and $\mathrm{JH}$ revised the protocol and manuscript critically for content. All authors approved the final version to be published and are accountable for all aspects of the work. NMC is the guarantor for the overall content.

Funding Dr McCaffrey is the recipient of a Victorian Government Mid-Career Research Fellowship through the Victorian Cancer Agency (MCRF20049) and Dr Lal is the recipient of a Victorian Government Early-Career Research Fellowship through the Victorian Cancer Agency (ECRF 20005). This review is also supported by the Economics of Cancer collaboration funded by Cancer Council Victoria. The funders had no role in the development of the protocol.

Competing interests None declared.

Patient consent for publication Not applicable.

Provenance and peer review Not commissioned; externally peer reviewed.

Supplemental material This content has been supplied by the author(s). It has not been vetted by BMJ Publishing Group Limited (BMJ) and may not have been peer-reviewed. Any opinions or recommendations discussed are solely those of the author(s) and are not endorsed by BMJ. BMJ disclaims all liability and responsibility arising from any reliance placed on the content. Where the content includes any translated material, BMJ does not warrant the accuracy and reliability of the translations (including but not limited to local regulations, clinical guidelines, terminology, drug names and drug dosages), and is not responsible for any error and/or omissions arising from translation and adaptation or otherwise.

Open access This is an open access article distributed in accordance with the Creative Commons Attribution Non Commercial (CC BY-NC 4.0) license, which permits others to distribute, remix, adapt, build upon this work non-commercially, and license their derivative works on different terms, provided the original work is properly cited, appropriate credit is given, any changes made indicated, and the use is non-commercial. See: http://creativecommons.org/licenses/by-nc/4.0/.

\section{ORCID iD}

Nikki McCaffrey http://orcid.org/0000-0003-3684-3723
REFERENCES

1 Samet JM. Tobacco smoking: the leading cause of preventable disease worldwide. Thorac Surg Clin 2013;23:103-12.

2 Benziger CP, Roth GA, Moran AE. The global burden of disease study and the preventable burden of ncd. Glob Heart 2016;11:393-7.

3 GBD 2015 Tobacco Collaborators. Smoking prevalence and attributable disease burden in 195 countries and territories, 19902015: a systematic analysis from the global burden of disease study 2015. Lancet 2017;389:1885-906.

4 World Health Organisation. WHO framework convention on. Geneva, Switzerland: Tobacco Control, 2003.

5 White S, McCaffrey, N, Scollo M. Tobacco dependence treatment in Australia - an untapped opportunity for reducing the smoking burden. Public Health Res Pract 2020;30:e3032020.

6 Clinical Oncology Society of Australia Smoking Cessation Working Group. Smoking cessation in cancer patients: embedding smoking cessation care in Australian oncology health services. Sydney, Australia: Clinical Oncology Society of Australia, 2020.

7 Nilan K, Raw M, McKeever TM, et al. Progress in implementation of who FCTC article 14 and its guidelines: a survey of tobacco dependence treatment provision in 142 countries. Addiction 2017;112:2023-31.

8 Shi Y, Warner DO. Surgery as a teachable moment for smoking cessation. Anesthesiology 2010;112:102-7.

9 Buchbinder M, Wilbur R, Zuskov D, et al. Teachable moments and missed opportunities for smoking cessation counseling in a hospital emergency department: a mixed-methods study of patient-provider communication. BMC Health Serv Res 2014;14:651.

10 Wiggers J, Vashum K, Wolfenden L, et al. Implementing nicotine dependence and smoking cessation care in hospitals: an evidence check rapid review brokered by the SAX Institute for the NSW Ministry of health, 2016. Available: https://www.saxinstitute.org.au/

11 Hutchinson J, Mangera Z, Searle L, et al. Treatment of tobacco dependence in UK hospitals: an observational study. Clin Med 2018;18:35-40.

12 Mullen KA, Walker KL, Hobler LA, et al. Performance obligations to improve delivery of Hospital-Initiated smoking cessation interventions: a before-and-after evaluation. Nicotine Tobacco Research 2021;23:77-84.

13 Care ACoSaQiH. Hospital-Aquired complications fact sheets. Sydney, NSW: Care ACoSaQiH, 2018.

14 Sørensen LT. Wound healing and infection in surgery. The clinical impact of smoking and smoking cessation: a systematic review and meta-analysis. Arch Surg 2012;147:373-83.

15 Bellomo R, Goldsmith D, Russell S, et al. Postoperative serious adverse events in a teaching hospital: a prospective study. Med $J$ Aust 2002;176:216-8.

16 Badia JM, Casey AL, Petrosillo N, et al. Impact of surgical site infection on healthcare costs and patient outcomes: a systematic review in six European countries. J Hosp Infect 2017;96:1-15.

17 Thomsen T, Villebro N, Møller AM. Interventions for preoperative smoking cessation. Cochrane Database Syst Rev 2014;3:CD002294.

18 Lee SM, Landry J, Jones PM, et al. Long-Term quit rates after a perioperative smoking cessation randomized controlled trial. Anesth Analg 2015;120:582-7.

19 Berlin NL, Cutter C, Battaglia C. Will preoperative smoking cessation programs generate long-term cessation? A systematic review and meta-analysis. Am J Manag Care 2015;21:e623-31.

20 Lee D, Lee Y-R, Oh I-H, . Cost-Effectiveness of smoking cessation programs for hospitalized patients: a systematic review. Eur $\mathrm{J}$ Health Econ 2019;20:1409-24.

21 Ruger JP, Lazar CM. Economic evaluation of pharmaco- and behavioral therapies for smoking cessation: a critical and systematic review of empirical research. Annu Rev Public Health 2012;33:279-305.

22 Berg ML, Cheung KL, Hiligsmann M, et al. Model-Based economic evaluations in smoking cessation and their transferability to new contexts: a systematic review. Addiction 2017;112:946-67.

23 Bolin K. Economic evaluation of smoking-cessation therapies: a critical and systematic review of simulation models. Pharmacoeconomics 2012;30:551-64.

24 McCaffrey N, Dowling G, White SL. The estimated effect of reducing the maternal smoking rate on neonatal intensive care unit costs in Victorian public hospitals. Aust Health Rev 2021;45:516.

25 McCaffrey N, Scollo M, Dean E, et al. What is the likely impact on surgical site infections in Australian hospitals if smoking rates are reduced? A cost analysis. PLoS One 2021;16:e0256424.

26 Al-Janabi H, McCaffrey N, Ratcliffe J. Carer preferences in economic evaluation and healthcare decision making. Patient 2013;6:235-9.

27 McCaffrey N, Cassel JB, Coast J. Bringing the economic cost of informal caregiving into focus. Palliat Med 2015;29:866-7. 
28 Merlo G, Page K, Ratcliffe J, et al. Bridging the gap: exploring the barriers to using economic evidence in healthcare decision making and strategies for improving uptake. Appl Health Econ Health Policy 2015;13:303-9.

29 Jiménez-Ruiz CA, Martín V, Alsina-Restoy X, et al. Cost-Benefit analysis of funding smoking cessation before surgery. Br J Surg 2020;107:978-94.

30 Moher D, Shamseer L, Clarke M, et al. Preferred reporting items for systematic review and meta-analysis protocols (PRISMA-P) 2015 statement. Syst Rev 2015;4:1-9.

31 Mills E, Eyawo O, Lockhart I, et al. Smoking cessation reduces postoperative complications: a systematic review and meta-analysis. Am J Med 2011;124:144-54.

32 Wong J, Lam DP, Abrishami A, et al. Short-Term preoperative smoking cessation and postoperative complications: a systematic review and meta-analysis. Can J Anaesth 2012;59:268-79.

33 Arber M, Glanville J, Isojarvi J, et al. Which databases should be used to identify studies for systematic reviews of economic evaluations? Int J Technol Assess Health Care 2018;34:547-54.

34 Covidence. Covidence systematic review software, veritas health innovation, Melbourne, Australia, 2014. Available: www.covidence.org

35 Watts RD, Li IW, . Use of checklists in reviews of health economic evaluations, 2010 to 2018. Value Health 2019;22:377-82.

36 Chiou C-F, Hay JW, Wallace JF, et al. Development and validation of a grading system for the quality of cost-effectiveness studies. Med Care 2003;41:32-44.

37 Le LK-D, Esturas AC, Mihalopoulos C, et al. Cost-Effectiveness evidence of mental health prevention and promotion interventions: a systematic review of economic evaluations. PLOS Med 2021;18:e1003606.

38 Husereau D, Drummond M, Petrou S, et al. Consolidated health economic evaluation reporting standards (cheers) statement. BMJ 2013;346:f1049.

39 Husereau D, Drummond M, Petrou S, et al. Consolidated health economic evaluation reporting standards (CHEERS)-explanation and elaboration: a report of the ISPOR health economic evaluation publication guidelines good reporting practices task force. Value Health 2013;16:231-50.

40 Ryan R. Cochrane consumers and communication review group: data synthesis and analysis. Cochrane Consumers and Communication Review Group 2013.

41 Jacobsen E, Boyers D, Avenell A. Challenges of systematic reviews of economic evaluations: a review of recent reviews and an obesity case study. Pharmacoeconomics 2020;38:259-67.

42 Rombey T, Eckhardt H, Quentin W. Cost-Effectiveness of prehabilitation prior to elective surgery compared to usual preoperative care: protocol for a systematic review of economic evaluations. BMJ Open 2020;10:e040262-e62.

43 Shemilt I, Thomas J, Morciano M. A web-based tool for adjusting costs to a specific target currency and price year. Evidence \& Policy: A Journal of Research, Debate and Practice 2010;6:51-9.

44 Engel L, Ajdukovic M, Bucholc J, et al. Valuation of informal care provided to people living with dementia: a systematic literature review. Value in Health 2021;19. 